\title{
N-Pattern Huffman Compression Algorithm for Medical Images in Telemedicine
}

\author{
Christy Sumitha Vincent ${ }^{1 *}$, Janet Jayaraj ${ }^{2}$ \\ ${ }^{1}$ Research Scholar, Manonmaniam Sundaranar University, Tirunelveli, India. \\ 2 Department of CSE,SVCET, Andhra Pradesh, India. \\ * Corresponding author. Tel: +919176722950; email: sumithathomasj@gmail.com \\ Manuscript submitted July 10, 2015; accepted November 20, 2015. \\ doi: $10.17706 /$ jcp.11.5.365-373
}

\begin{abstract}
The main purpose of this paper is to put forth an idea to reduce the size of the medical images to be transmitted optimally and qualitatively with the special focus in the field of telemedicine. The fact that the bandwidth factor influences the data transfer rate, the data has to be as small as possible to be transmitted and downloaded swiftly across the nets. A new method has to be proposed, where by the compression of the data could be still improved. This has been achieved by improving the existing Huffman lossless compression algorithm. The proposed N-Pattern Technique focuses on identification and grouping the different length patterns based on their intensity values and optimizing the input to the encoder for compression. On comparison with the existing method, the proposed method greatly improves the compression and the space saving with the quality of the image being still maintained.
\end{abstract}

Key words: Data compression, entropy, Huffman coding, mean squared error (MSE), N-pattern-sequencer, peak signal-to-noise ratio (PSNR), telemedicine.

\section{Introduction}

In the last decades, there has been a tremendous growth in the innovative technologies, which were based on the communication technology and has revolutionized the humanity. There are a number of laws and observed principles of the earlier decades that shape the way that technology evolves and is able to evolve. The very famous Moore's Law states that the processing power of a microchip doubles every eighteen months meaning that the computers become faster and the price of computing power halves every eighteen months. The Gilder's Law states that the total bandwidth of communication systems triples every twelve months. The Metcalfe's Law states that the value of a network is proportional to the square of the number of nodes, meaning that as the network grows, the value of being connected to it also grows exponentially [1]. Connectedness is the key objective in the modern life and society. Now being connected, there is a large potential of benefits that can be achieved by proper coordination of technologies. The deployment of high speed networks and reduction in bandwidth requirements has led to the emergence of diverse technological applications. Telemedicine is one such technology which has a very high potential of applications for the present and the future society. It bridges gap between doctors and patients, thereby, overcoming the barriers of distance and time which deals with a very huge volume of data [2]. Though the computational speeds have improved a lot in the last decades, still there is a significant lag when it comes to the bandwidth due to the Shannon's limit, which states that there is a limit to the amount of information that can be transmitted through a mobile radio channel where by the maximum gain of three times is achievable for third generation 
systems. Moreover, it is a well known fact that the radio signals get attenuated or reflected by most obstacles and at the higher frequency ranges, the diffraction and reflection losses do get increased [3]. Hence the demand for bandwidth goes high and the optimized use of the bandwidth is a must. Data compression techniques as such can be divided into two major families namely lossy and lossless. Lossy data compression concedes a certain loss of accuracy in exchange for greatly increased compression. Since telemedicine deals with high volume of medical images, mostly loss is not acceptable. A lossless compression algorithm is needed which guaranties to generate an exact output of the input data stream after a compress or expand cycle [4]. Among many diagnostic emergencies, there are also time critical diseases like stroke, where each seconds the brain cells die, a rapid access to the medical service is important [5]. Obviously, in such a scenario the compressed data will enable the fast transmission of data even with limitations of the bandwidth. So, more effective methods have to be applied for attaining maximum compression of data to fit to the bandwidth without compromising with the quality [4]. A comparitive study between Huffman and Arithmetic codings has been consolidated and proved that from the implementation point of view, Huffman coding is easier and need less execution time than the arithmetic coding [6]. Arithmetic algorithm yields much more compression ratio than Huffman algorithm, which means that in some applications where the the time is not so important, the arithmetic algorithm can be applied to achieve high compression ratio. But on the contrary, for the real time applications that are time oriented, the Huffman algorithm is recommended. A lossless compression method has been demonstrated based on wavelet decomposition and correlation analysis of coefficients for the medical MRI and CT images [7]. An improved Huffman compression algorithm has been introduced by the reduction of the source symbols and the improvement in the compression has been proved [8]. An effective compression algorithm has been proposed which is based on Huffman coding but with the application of the technique called pattern finder [9]. The operation of the pattern finder is to search out the most effective pattern that is the most frequent occurring pattern. Furthermore, an improved three pattern Huffman compression algorithm has been extended for three best patterns and improvement in the compression ratio has been depicted [10]. However, as the number of best pattern search increases above five, there is increase of complexity in pattern search and degradation in the image quality [2]. Based on the above mentioned constraints, there is still a potential for improvement to address the issues. So, a new method called N-Pattern sequencer technique has been proposed.

\section{Proposed Method}

Telemedicine is one of the emerging fields which deals with the huge amount of medical images and data. Practically, compression of the images is of importance for quickly transmitting the data. In the medical field the compression should be lossless. So increasing the compression ratio of the medical images without any loss is still a challenging topic. It is well known fact that the compression ratio and the storage space are inversely proportional. Hence, increasing the compression ratio decreases the storage space [9]. Less the data size, faster is the transmission speed and download speed. So a new approach has to be found where by the better compression is achieved than the existing techniques.

\subsection{Overview}

The idea behind the proposed method is to remove the redundant patterns from the image as much as possible and the resultant being sent to the Huffman encoder for further compression. Due to this pre-removal of the patterns, the number of code length used to represent those patterns are reduced. This pre-processing can be attributed to a significant increase in the compression ratio. The proposed method basically uses the most popular Huffman encoding due to its simplicity and the computational speed.

The Huffman code creates variable length codes that are an integral number of bits. Symbols with the higher probabilities get shorter code. Huffman codes have the unique prefix attribute, which means they can 
be correctly decoded despite being of variable length [4].

The basic steps involved in constructing the tree are:

Step 1: Sort the frequency table in the descending order.

Step 2: Identify the two symbols with the lowest frequencies and combine them to create a parent node. The identified symbols frequencies are added and the value is assigned to the parent node.

Step 3: Label each of the branch in the constructed tree with 0's and 1's.

Step 4: Repeat the above mentioned steps until only one free node is left.

The overview of the new method is shown in the Fig. 1. The optimization is achieved by the $\mathrm{N}$-Pattern-Sequencer. The importance of the pattern sequencer is to group the various patterns. The major grouping is based on the repetitive and non-repetitive patterns. Further division is based on the varying lengths of the patterns and their corresponding frequencies of appearances. Finally, the most frequently appearing patterns of selective lengths are taken as input to group their sub-patterns, which leads to optimize the input data for the Huffman process. When compared to the existing Huffman process, the optimized input enhances the compression ratio significantly.

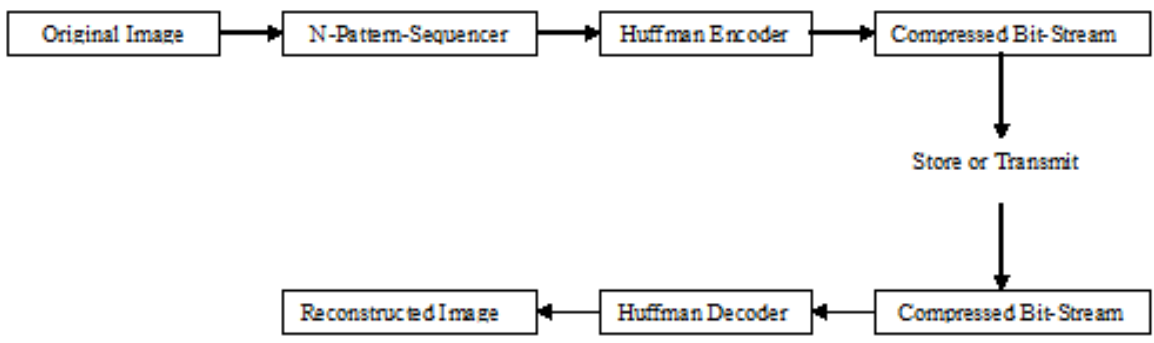

Fig. 1. Overview of the proposed N-pattern method.

\subsection{N-Pattern-Sequencer Technique}

In this technique, the main activity is the identification and grouping of the repetitive and non-repetitive patterns for the various intensity values of the Images.

Step 1: As shown in Fig. 2, the patterns are being identified and grouped as repetitive patterns which have identical intensity values and as the non-repetitive patterns which have the non-identical intensity values.

Step 2: Each grouped patterns are stored with their counts of occurrences.

Step 3: The grouped patterns are sorted in the descending order based on their counts of occurrences in order to trace the best patterns, where the best patterns refers to the most frequently occurring patterns.

Step 4: Each best patterns of various length (n) also have their own corresponding sub-patterns whose length should be minimum 2 .

Example: 5467

$\{546\}\{467\}\{54\}\{46\}\{67\}$

Step 5: The original image is searched for the best patterns and their corresponding sub-patterns whose positional information's are stored, whose accuracy is important for the decoder.

Step 6: The optimization is done by removing all the best patterns and their corresponding sub-patterns from the image.

Step 7: Optimized image data is being given as an input to the Huffman-encoder, where the output of the encoder is the compressed data.

The Percentage (\%) of compression ratio is calculated by using the following formula [9].

$$
\% \text { of compression ratio }=\frac{(\text { Originalfile size })-(\text { Compressedfile size })}{(\text { Original file size })} \times 100
$$


Step 8: The compressed data from the Huffman encoder is being sent to the Huffman decoder. To the decoders output, the best patterns and their corresponding sub-patterns are re-inserted by using their stored positional information for the reconstruction of the image.

Step 9: Checking for the quality of the original and the reconstructed images are done by calculation of the Mean Squared error and Entropy values.

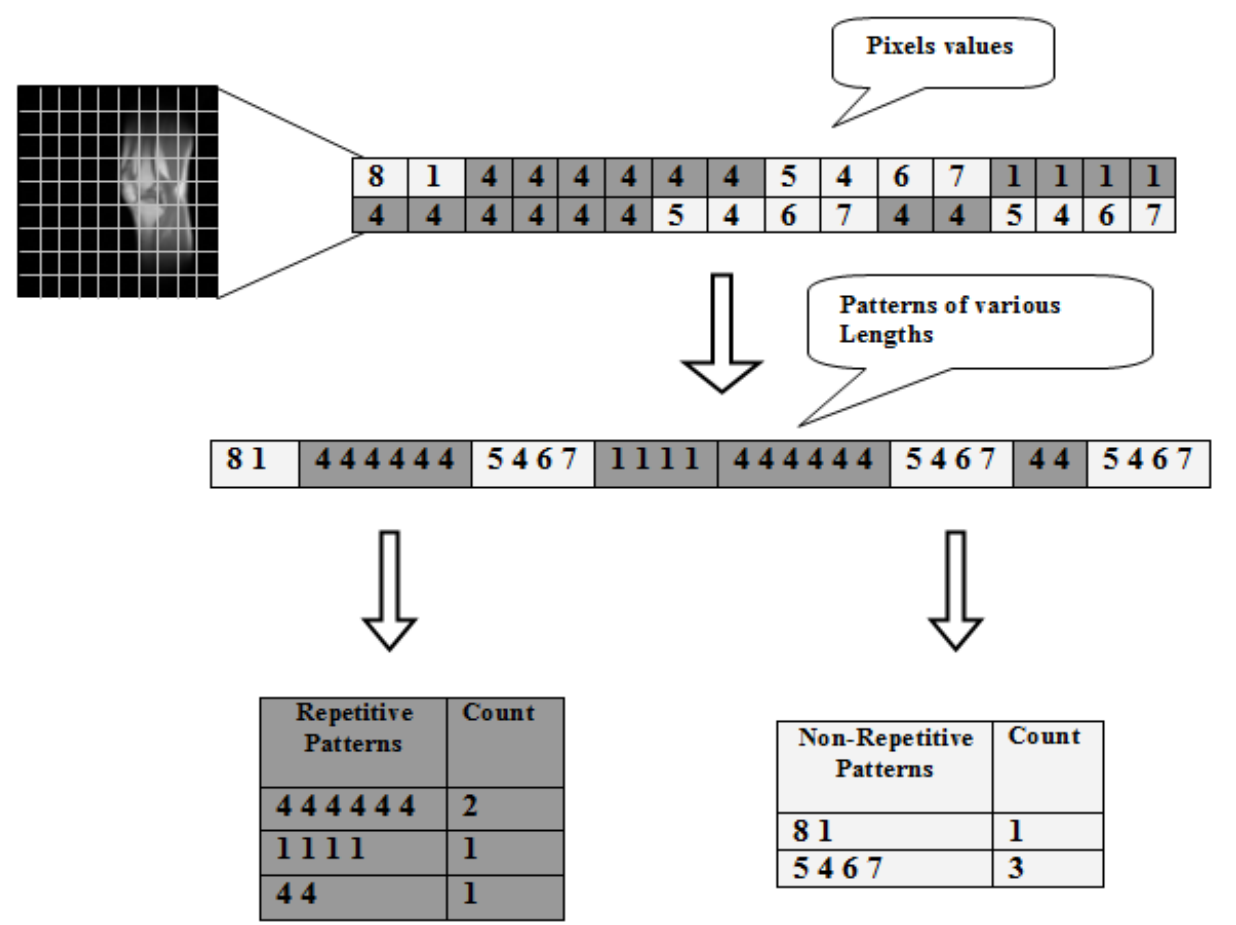

Fig. 2. Grouping of the repetitive and non-repetitive patterns.

\section{Quality Metrics for Reconstructed Image}

There are many methods to study about the quality of the reconstructed images. Among them, Entropy, MSE and PSNR are being applied.

\subsection{Entropy}

The entropy is a measure that tells how many bits are needed to code the image data, whose formula is represented below.

$$
\text { Entropy }=-\sum_{g=0}^{L=1} P(g) \log _{2}[P(g)]
$$

where, $L$ is the number of gray levels and $P(g)$ is the probability associated with gray level $g$ [11].

More the distribution due to gray levels, higher is the entropy associated. Moreover entropy of an image is also a measure of how much an image can be compressed without losing any information in that image. As a corollary, a compressed image that is maximally compressed, without losing any information, establishes the entropy of that. image [12]. It implies that if the entropies of original image and the reconstructed image are equal, then the images are identical.

\subsection{Mean Squared Error(MSE)}

The MSE represents the cumulative squared error between the compressed and the original image, whose formula is represented below. 


$$
M S E=\frac{1}{m n} \sum_{0}^{m-1} \sum_{0}^{n-1}\|f(i, j)-g(i, j)\|^{2}
$$

where,

$f:$ the matrix data of original image

$g$ : the matrix data of reconstructed image

$m$ : the numbers of rows of pixels

$n$ : the number of columns of pixels

$i, j$ : refers to rows and columns index

\subsection{Peak Signal-to-Noise Ratio(PSNR)}

PSNR represents a measure of the peak error. The lower the value of MSE, lower the error and higher the PSNR value , the better the quality of the reconstructed image [13], whose formula is represented below.

$$
P S N R=20 \log _{10}\left(M A X_{f} / \sqrt{M S E}\right)
$$

where, $M A X_{f}$ is the maximum signal value that exists in the original image.

\section{Experimental Results and Discussions}

The proposed method has been applied on twenty five sets of various medical imaging data which includes MRI, CT and Ultrasound images with the file extensions of $\mathrm{dcm}$, jpeg and bmp respectively. Three sample images have been tested with the new method where Sample 1 is a MRI (Knee1.dcm), Sample 2 is a CT (Brain.bmp), and Sample 3 is an Ultrasound (CarotidArtery.jpg). The N-Pattern-Sequencer groups the patterns into two major division namely repetitive and non-repetitive patterns. The Fig. 3 shows the corresponding results for Sample 1. It is obvious from the graph that the percentage of compression for repetitive patterns are less than the non-repetitive patterns for a pattern length $(n=5)$.

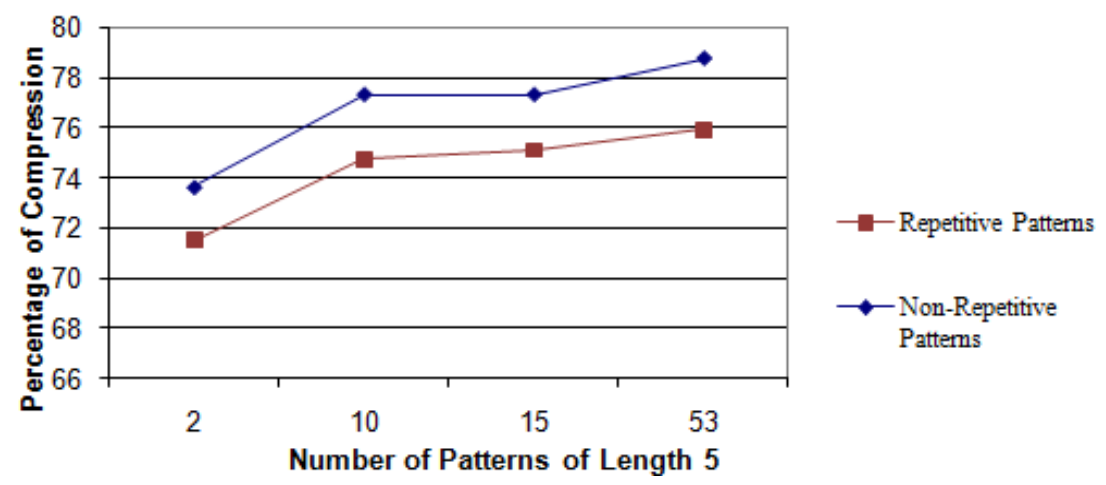

Fig. 3. Comparison of repetitive and non-repetitive patterns for sample 1.

The considered frequencies for the patterns are 2,10,15 and 53, where it has been noted that in the case of repetitive patterns, the maximum appearance of the length $(n=5)$ pattern is 53 . Whereas, in the case of non-repetitive patterns, the maximum appearance of the length $(n=5)$ pattern is 929 . It has been noted that the appearance of non-repetitive patterns is always greater than the repetitive patterns and which will give more ranges of sub-patterns. Therefore, further analysis is more focused on the non-repetitive patterns. Further, Fig. 4 shows the effect of the N-Pattern Technique based on Sample 1.

In this case, the original image occupies 524288 bytes. The Huffman technique reduced the image to 163960 bytes. The N-Pattern Technique still reduces the image size to 73756 bytes which means 90204 
bytes difference with respect to Huffman technique which is significantly important. Further the Sample 1 was analysed based on variation in the length and frequency of patterns, whose results are tabulated in the Table 1 and Table 2.

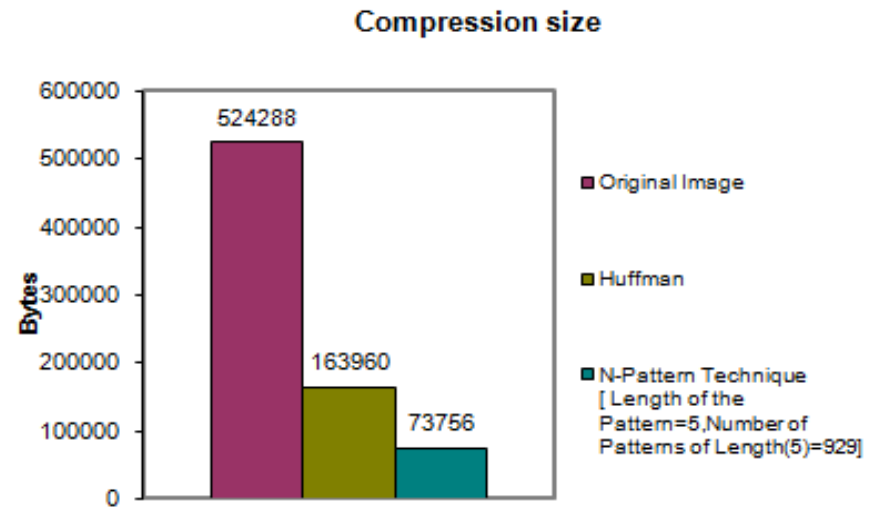

Fig. 4. Effect of Huffman and N-Pattern technique on sample 1.

Table 1. Compression Results for the Patterns of Length $n=5$

\begin{tabular}{lccccc}
\hline \hline Technique & $\begin{array}{c}\text { Length of the } \\
\text { Pattern }(n)\end{array}$ & $\begin{array}{c}\text { No.of Patterns of } \\
\text { length }(n)\end{array}$ & $\begin{array}{c}\text { No.of bytes } \\
\text { reduced }\end{array}$ & $\begin{array}{c}\text { Compression } \\
\text { Ratio }\end{array}$ & $\begin{array}{c}\text { Compression } \\
\text { Percentage \% }\end{array}$ \\
\hline Huffman & ----- & ----- & 360328 & 3.19 & 68.72 \\
N-Pattern & 5 & 2 & 386140 & 3.79 & 73.65 \\
N-Pattern & 5 & 10 & 405461 & 4.41 & 77.33 \\
N-Pattern & 5 & 400 & 428086 & 5.44 & 81.65 \\
N-Pattern & 5 & 929 & 450532 & 7.10 & 85.93 \\
\hline \hline
\end{tabular}

Table 2. Compression Results for the Variation Length of the Patterns

\begin{tabular}{lccccc}
\hline \hline Technique & $\begin{array}{c}\text { Length of the } \\
\text { Pattern }(n)\end{array}$ & $\begin{array}{c}\text { No.of Patterns of } \\
\text { length }(n)\end{array}$ & $\begin{array}{l}\text { No.of bytes } \\
\text { reduced }\end{array}$ & $\begin{array}{c}\text { Compression } \\
\text { Ratio }\end{array}$ & $\begin{array}{c}\text { Compression } \\
\text { Percentage \% }\end{array}$ \\
\hline Huffman & ------ & ----- & 360328 & 3.19 & 68.72 \\
N-Pattern & 5 & 10 & 405461 & 4.41 & 77.33 \\
N-Pattern & 10 & 10 & 411514 & 4.45 & 78.49 \\
N-Pattern & 20 & 10 & 419221 & 4.99 & 79.96 \\
N-Pattern & 30 & 10 & 418388 & 4.95 & 79.80 \\
\hline \hline
\end{tabular}

The Sample 1 was an MRI image of size $512 \times 512$ and the Huffman method gave a compression ratio of 3.19 with a space saving of $68.72 \%$. Comparatively, the proposed new method for a pattern length of $n=5$ and frequency 929 which is the corresponding maximum appearance, gave compression ratio of 7.10 with a space saving $85.93 \%$ shows an increased saving by 17.21 percent.

Furthermore, the proposed method is tested for various patterns of length $n=5,10,20,30$ but with the fixed frequency of 10 and the results are tabulated in Table 2. In the Table 1, the values show that for less pattern length and increasing frequency, the compression ratio keeps increasing. Where as in the Table 2, the values show that as the pattern length increases, the compression ratio gradually increases and later gets decreased. The reason for this behaviour is that the frequencies of such patterns are less. Nevertheless, in both the cases, the proposed method gives better percentage of compression than the existing Huffman method. A model output of the N-Pattern Technique for sample 1 is shown in Fig. 5, where the graphical user interface gives the flexibility to analyse various images with various parameters of interest. Since the results 
were appealing, the proposed method has been applied on the other common types of medical imaging data like CT and Ultrasound images.

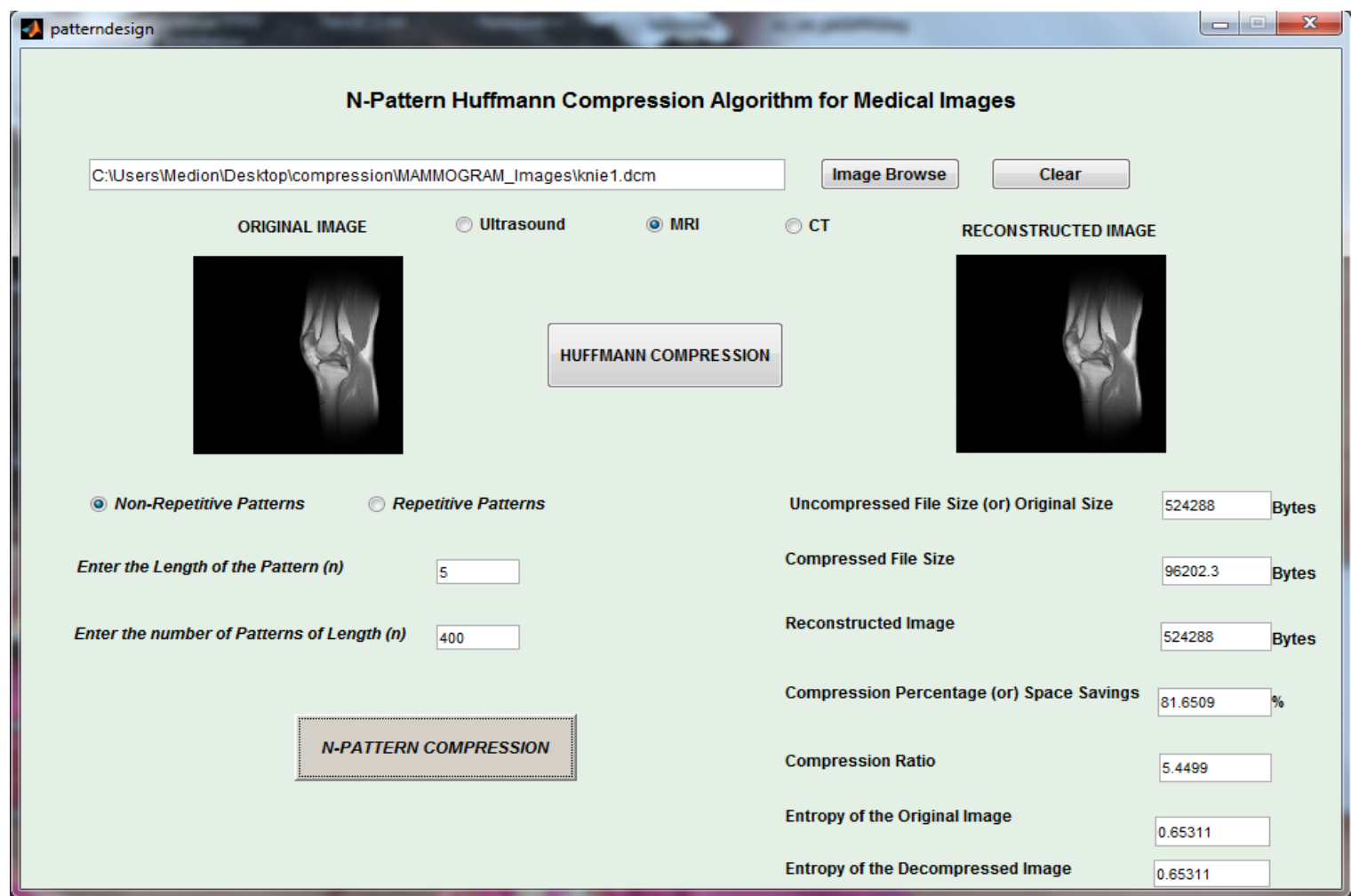

Fig. 5. N-Pattern technique for sample 1.

The compression achieved in MRI, CT and Ultrasound Images for various lengths of patterns but with the fixed frequency of 10 is being depicted in Fig. 6. Comparison of the illustrated results show that as the pattern length increases, the compression percentage gets either increased or maintained.

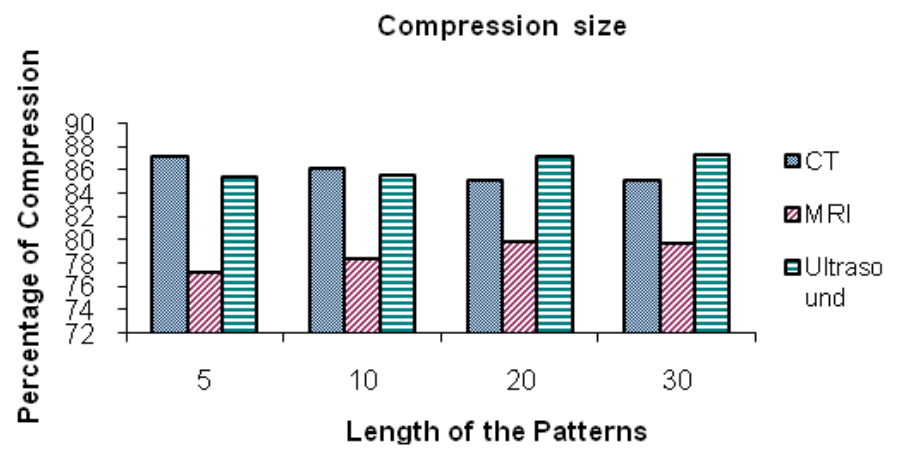

Fig. 6. Effect of N-Pattern technique for the variation length of the patterns on sample 1, sample 2 and sample 3.

The above results leads to the further analysis and is observed that improvement in the compression is purely dependent on the frequency of the occurring patterns. More the number of times the pattern appears, the more is the compression possible. Moreover, based on the various images analysed, it could be concluded that smaller the length of the patterns and larger their frequencies, higher is their compression.

In the proposed method, the quality of the images are being analysed by the terms of Entropy, MSE and PSNR. The entropy value for the original image and the reconstructed image are the same for which MSE is zero and PSNR is infinite. It means that there is no difference between original and the reconstructed image. 
This also confirms that there was no loss of data.

\section{Conclusion}

The existing 2-3 pattern method has its own limitations of pattern length, quality and compression improvement of 4-5\% over Huffman has been depicted [2]. Comparitively, the proposed "N-Pattern Technique" shows a significant improvement in the compression ratio of $14-17 \%$ and the space saving compared to the existing Huffman method. The method also is applicable for more common extensions of medical images together with a flexibility of pattern length. The metrics of the Entropy and the PSNR confirms with the lossless quality. The results also show that the compression ratio of the images considered depends on the length of the non-repetitive patterns and its frequency of appearance. For lesser the length of the pattern and higher their frequencies, larger is the compression ratio. It also shows that for length of the patterns of range ( $n=5$ to 20 ) gives better compression. Obviously the future will be driven by compact instruments and their connectivity bringing more facility for the humanity. So the better compression techniques have high potential and market in telemedicine applications especially for the nations that have still a limited coverage or bandwidths for communication. Hence the proposed method can be further refined and optimized for videos. More the interconnection of the medical devices and their related technologies, the risks associated cyber-security also grows exponentially. So the future work could also be extended with such combined fields. As Image based technologies have ever growing scope in the new applications, the compression techniques associated with it has much importance due to the bandwidth constraints associated with it.

\section{References}

[1] Pinto, J. (2003). Automation Unplugged. North Carolina: ISA.

[2] Janet, J., Divya, M., \& Meenalosini, S. (2011). Lossless compression techniques for medical images in Telemedicine. In G. Graschew, \& T. A. Roelofs (Eds.), Advances in Telemedicine Technologies, Enabling Factors, Scenario (pp. 111-130). Austria: Intech.

[3] Webb, W. (2001). The Future of Wireless Communications. Norwood: Artech House.

[4] Nelson, M., \& Gailly, J. L. (1995). The Data Compression. New York: M and T Books.

[5] Mullen, M. T., et al (2015). Optimization modeling to maximize population access to comprehensive stroke centers. American Academy of Neurology, 84(12), 1196-1205.

[6] Shahbahrami, A., Bahrampour, R., Rostami, M. S., \& Mobarhan, M. A. (2011). Evaluation of huffman and arithmetic algorithms for multimedia compression standards. International Journal of Computer Science, Engineering and Applications, 1(4), 34-47.

[7] Ramesh, S. M., \& Shanmugam, A. (2010). Medical image compression using wavelet decomposition for prediction method. International Journal of Computer Science and Information Security, 7(1), 262-265.

[8] Saravanan, C., \& Ponalagusamy, R. (2009). Lossless grey-scale image compression using source symbols reduction and Huffman coding. International Journal of Image Processing, 3(5), 184-251.

[9] Janet, J., \& Natesan, T. R. (2005). Effective compression algorithm for medical images as an aid to Telemedicine. Asian Journal of Information Technology, 4(12), 1180-1186.

[10] Mohandass, D., \& Janet, J. (2010). An improved three pattern huffman compression algorithm for medical images in telemedicine. In V. V. Das, et al. (Eds.), Proceedings of the International Conference on Business Administration And Information Processing (pp. 263-268). Springer-Verlag Berlin Heidelberg.

[11] Umbaugh, S. E. (2005). Computer Imaging: Digital Image Analysis and Processing. Florida: CRC Press Book.

[12] Ali, S., Graff, C., \& Morris, D. S. (2005). Concepts for entropy-aware image transport over 
energy-limited, ad-hoc networks. Proceedings of 2005 IEEE/Sarnoff Symposium on Advances in Wired and Wireless Communication (pp. 9-12). Princeton, NJ:IEEE.

[13] Kumar, T., \& Kumar, R. (2015). Medical image compression using hybrid techniques of DWT,DCT and Huffman coding. International Journal of Innovative Research in Electrical, Electronics, Instrumentation and Control Engineering, 3(2), 54-60.

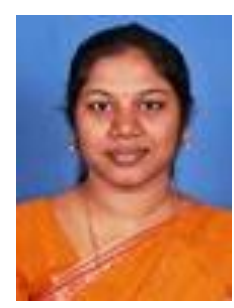

Christy Sumitha Vincent was born in Chennai, India. She received her M.C.A degree from Madurai Kamaraj University, Madurai, India in 2006 and M.Phil in computer science from Manonmaniam Sundaranar University, Tirunelveli, India in 2007. She is currently pursuing her research in medical image processing under the guidance of Professor Dr. Janet Jayaraj.

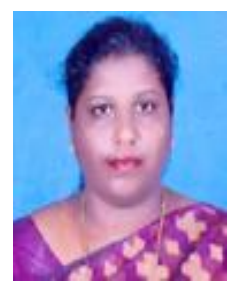

Janet Jayaraj received her B.E and M.E degrees in computer science \& engineering from Madras University. She has completed her Ph.D. from Sathyabama University, Chennai in 2006. Currently she is working as a professor and the head of the Department of Computer Science \& Engineering in Sri Venkateswara College Of Engineering \& Technology, Chitoor, Andhra Pradesh, India. She has received Young Scientist recognition for her DST project, 'Optimizing Data movement in Cloud'. Until now, She has produced 7 doctorates. She is presently guiding 5 doctoral candidates. She has received several research funds to the tune of 30 Lakhs from Government of India for her research projects. Her research interests include image processing, data mining and wireless sensor networks. 